\title{
AN EVALUATION MODEL OF TECHINING ASSISTANT USING ARTIFICIAL NEURAL NETWORK
}

\begin{abstract}
=
AHMED HAMZA OSMAN ${ }^{1}$

${ }^{1}$ Department of Information System, Faculty of Computing and Information

Technology, King Abdulaziz University, Jeddah, Kingdom of Saudi Arabia

*Correspondence: ahoahmad@kau.edu.sa

ABSTRACT. This study presents an efficient framework for assessment and prediction of teachers' performance in academic institutes using Artificial Neural Network (ANN) algorithm. The prediction model was tested effectively using the TA UCI dataset. The data consists of academic experiences for teachers as well as their experiences and grades of students in courses they taught among others. The SPSS tool was used to build the suggested prediction system. The TA data was dividing into three groups (70, 80, and 90) for training data, and (30,20, and 10) for testing data respectively to study the dataset discrimination. The results are showing that the neural network obtained better accuracy results with (90\%) in the training and $(10 \%)$ in testing.
\end{abstract}

Keywords: Teaching Assistant; classification; Performance, Neural Network; accuracy.

1. Introduction. Nowadays, most of the academic institutes facing a low-quality problem in the educational field. One of these factors is an educational student achievement and staff teaching quality. Some studies had been done to engorge the students to improve their academic achievement, but still, the problem of the teaching quality need to be improved especially in the practical parts that normally performed by the Teaching Assistant staff and Lecturer. Teaching Assistants (TAs) are persons who help lecturer or professors with instructional tasks [1] and [2]. TAs can be in university level, secondary school level or elementary school level. Evaluation Teaching Assistant system introduced in agreement with the Rules as a recommendation from the Evaluation and Standards Uniform Performance Teacher as one of the improvement factor of learning quality [3]. Performance evaluation system assists the district's scholar attainment aims and the universities as well. This program offers us efficiency instructive environment in the education institutes. It is permitting for ingenuity and separate instructor initiative. The efficiency of the teachers may be effect in some courses because of teaching language, study time, the number of students in each classroom, etc. The aim is to assist the incessant growing and development progress of every instructor by analyzing, monitoring, and employing relevant information amassed within a model of meaningful reaction to enhance the progress of academic scholar and instructor efficiency. The significant objective of this study is to the proposal a solid evaluation system for TA using on Artificial Neural Network algorithm (ANN). The proposed model applied the ANN as a possible solution to study and analyze the quality of teaching assistant teachers to improve their skills to growth the educational achievement level. This study is systematized as follows. Section 2 discuss the related studies. In Section 3 the proposed model using neural network algorithm is explained. The dataset and experimental design are organized and discussed in Section 4. Results and discussion behind this study are clarified in Section 5, while Section 6 is a conclusion of this paper.

2. Related Work. There are many studies has been done to improve the quality of TA staff. The improving performed in the curriculum and the educational development issues. Baradwaj and Pal [4] conducted the task of prediction to assess the performance of students in the academic field. The decision tree classifier technique utilized for classifying the student performance. Thier proposed method extracted new information that can define scholars' performance level in last semester examination. It supports previously in finding the 
failures and scholars who required superior care to decrease failure portion and let the instructor offer suitable guiding to take proper action for the coming semester examination. The C4.5 and CART decision tree classification models have been used by Yadav and Pal [5] on engineering scholar's dataset to predict their achievement in the final exams. These classification models contain psychological, social, and other environmental parameters required for the actual classification of the student's achievement. The C4.5 algorithm has obtained better performance compared with CART technique. The true positive degree of their models achieved FAIL class with 0.786 for the $\mathrm{C} 4.5$ classifier. Their models can classify the new scholars in short time but the prediction accuracy list for the scholars by employing the prediction systems to the records of arriving new scholars. Ola and Pallaniappan [6] utilized a smart technology-oriented model to assess the performance of teachers in higher education institutions and introduced a good method for designing appropriate system framework to predict the performance of teachers. The introduced method is fully applied to help school administrators to make decisions and providing the basis for teachers' performance enhancement that will achieve the best academic results for students and enhance the standard teaching. Thus, this will support to the effective teaching attainment. Ahmadi and abadi examined [7] the instructor evaluation performance of final semester of an academy and presented the results which are obtained using WEKA software. They used 104 instructor's activities samples in the classroom with machine learning techniques such decision tree methods and association rule algorithms. Mardikyan and Badur [2] introduced a TA evaluation system based on two types of data mining methods, decision trees, and regression. Their research objective focused on comprehending the main factors influencing the learning performance of the teachers by defining the associated factors with the learning performance from 2004 to 2009. The work position of the teachers that is not involved in the survey is found to be important. The main significant factor to clarify the teachers' learning performance is the teacher arrogances that are mainly evaluated by the performance steps. The teachers that appeal more scholars to the courses assessed more positively.

3. Proposed model. The research design of this study is a combination of several stages. This study consists of 6 stages. Each stage contains a different number of steps. Firstly, TA data collection from (UCI). While the second phase of the data for training and the other for testing, and the third phase is defining the data field, input and output, and the fourth phase we will use a neural network that represents a sample and data accuracy algorithm. The fifth phase will represent the results in graphical shape. The sixth stage is research writing. The structure of the introduced system as demonstrated in figure 1.

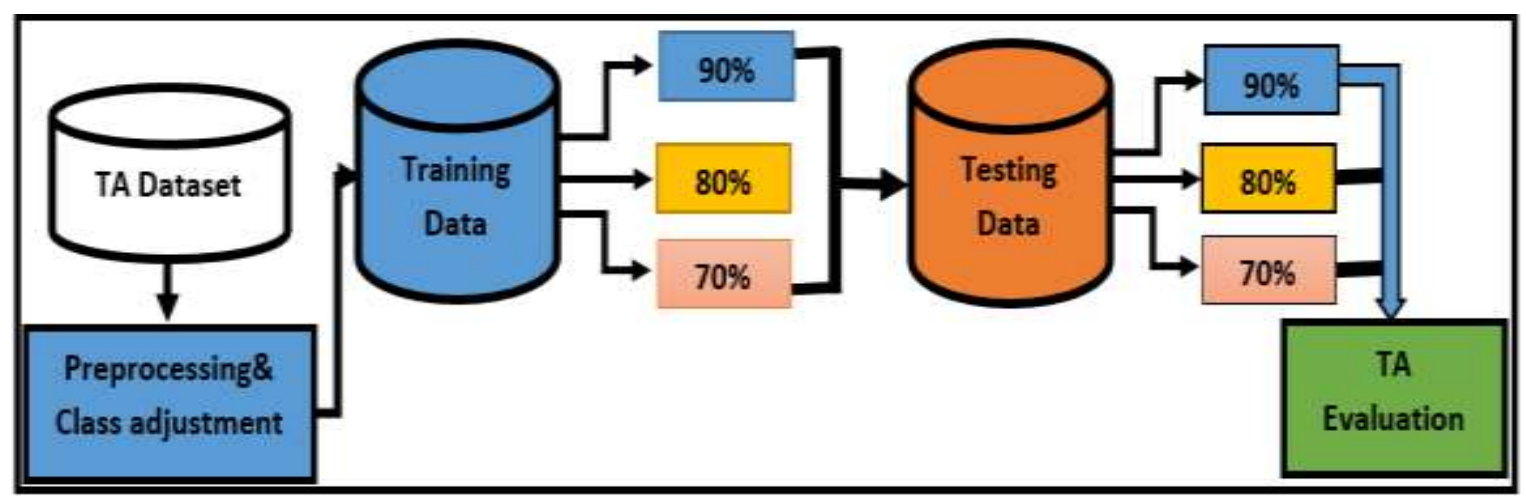

Figure 1. Proposed Model

The structure of the introduced system as demonstrated in figure1. Firstly, the preprocessing and class adjustment step adopted in the input and output features. The first five features employed as an input layer of the NN model. However, the last feature (class) used as an output feature of the NN. The majority of the $\mathrm{NN}$ algorithms used as a binary classifier in the target feature ( 0 and 1$)$. However, the current TA dataset used as a non-binary class in the target field $(1,2,3)$. The proposed study adopted the target feature (class) to be binary (0/1) to work efficiently. In this study, the class (2) is modified into class (1) and class (3) to be a binary class. The experiments have done after the modification and adaptation of the target field. The teaching assistant evaluation dataset then divided into different data groups such as $90-10 \%, 80-20 \%$ and $70-30 \%$ training and testing stages. These partitions are performed on the proposed model using ANN. The 
performance in all partitions calculated regarding accuracy, execution time and misclassification error.

4. Dataset and Experimental Design. The TA dataset [8] contain assessments of teaching outcomes over two summer semesters and three regular semesters of 151 TA staff assignments at the Department of Statistics in the Wisconsin-Madison University. The target field was categorized into three equal-sized classes "1, 2, and 3" for "Low, High, and Medium" respectively. The data contains descriptions assessments of teaching Dataset which can classify the quality of their teaching assistant staff according to TA staff performance. The number of features is five includes; TA language, Course, Course Instructor, Class size, and Teaching Time. Different studies in teaching assistant evaluation used the TA UCI dataset such as [9] and [10].Table 1 demonstrates the TA dataset features.

Table 1: The TA dataset features

\begin{tabular}{|c|c|l|}
\hline Attributes Name & Attributes Type & Attributes Possible Values \\
\hline English_speaker & Binary & $\begin{array}{l}\text { 1=English speaker, } \\
2=\text { non English speaker }\end{array}$ \\
\hline Course_instructor & Categorical & 25 category \\
\hline Course & Categorical & 26 category \\
\hline Regular_semester & Binary & $\begin{array}{l}\text { 1=summer semester } \\
2=\text { Regular semester }\end{array}$ \\
\hline Class_size & real & \\
\hline Class_attributes & & $1=$ Low \\
& & $2=$ Medium \\
& & $3=$ High \\
\hline
\end{tabular}

The section discusses the experiment, results, and discussion about the introduced model. The proposed model used the SPSS software to the classifier and predicted the TA staff performance. The SPSS software is a statistic tool and recently used in data mining problems. The tool adopted by combined different types of machine learning algorithms to solve the prediction challenges in a different file. It can suggest various modeling algorithms, such as segmentation used by Gaemperli and et.al[11], association detection applied by Jorge and Azevedo [12], and classification techniques adopted in different areas such as [13], [14], and [15]. The neural network adopted on the data set for the input and output features as shown in figure 2 .

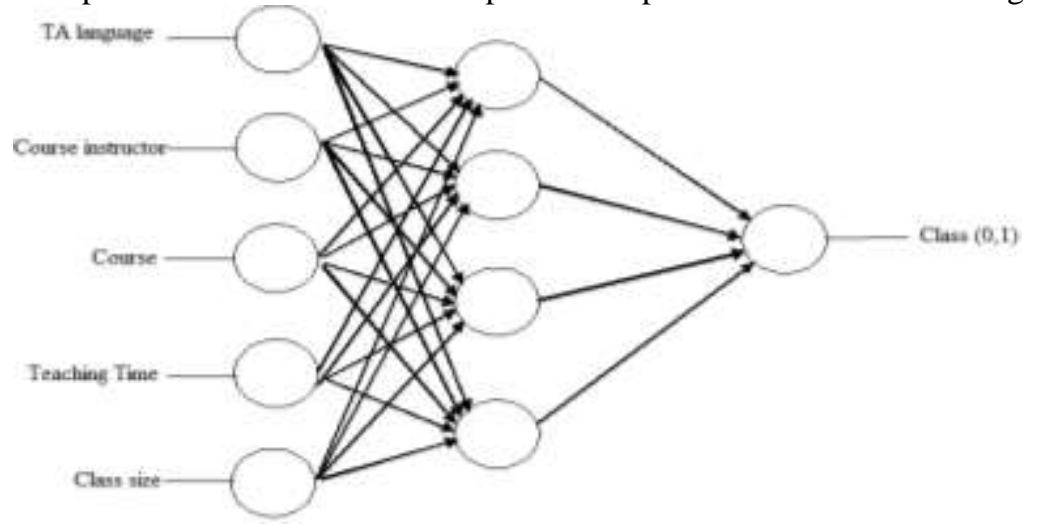

Figure 2. The TA Neural Network Model

By using the SPSS tool, the TA dataset divided into $90 \%, 80 \%$, and $70 \%$ for training and $10 \%, 20 \%$, and $30 \%$ for testing data respectively. Table 2 shown the training and testing dataset.

Table2. Training and testing Dataset

\begin{tabular}{|c|c|c|c|}
\hline Groups & Size1 & Size2 & Size3 \\
\hline Training & $90 \%(136)$ & $80 \%(121)$ & $70 \%(106)$ \\
\hline Testing & $10 \%(15)$ & $20 \%(30)$ & $30 \%(45)$ \\
\hline
\end{tabular}


5. Results and Discussion. The experiments of the proposed model achieved the different shape of results in training and testing stages. By using these results, the performance of the TA staff can be analyzed and controlled. The performance output is analyzed based on different factors such as accuracy, estimation time, and prediction error. The accuracy TA performance calculated as:

Where,

$$
\text { Accuracy }=\frac{(\mathrm{TN}+\mathrm{TP})}{(\mathrm{TN}+\mathrm{FP})+(\mathrm{TP}+\mathrm{FN})}
$$

TP is a True Positive; FP is a False Positive; TN is a True Negative; FN is a False Negative

The training and testing results illustrated in table3.

Table 3: Training and testing results

\begin{tabular}{|c|c|c|c|c|c|c|c|}
\hline $\begin{array}{c}\text { Training } \\
\text { Size }\end{array}$ & $\begin{array}{c}\text { Training } \\
\text { Accuracy }\end{array}$ & $\begin{array}{c}\text { Training } \\
\text { Error }\end{array}$ & $\begin{array}{c}\text { Training } \\
\text { Execution } \\
\text { Time }\end{array}$ & $\begin{array}{c}\text { Testing } \\
\text { Size }\end{array}$ & $\begin{array}{c}\text { Testing } \\
\text { Accuracy }\end{array}$ & $\begin{array}{c}\text { Testing } \\
\text { Error }\end{array}$ & $\begin{array}{c}\text { Testing } \\
\text { Execution } \\
\text { Time }\end{array}$ \\
\hline $70 \%$ & $97.283 \%$ & $2.72 \%$ & $21 \mathrm{sec}$ & $30 \%$ & $95.24 \%$ & $4.76 \%$ & $1 \mathrm{sec}$ \\
\hline $80 \%$ & $94.215 \%$ & $5.79 \%$ & $24 \mathrm{sec}$ & $20 \%$ & $90.00 \%$ & $10.00 \%$ & $2 \mathrm{sec}$ \\
\hline $90 \%$ & $96.324 \%$ & $3.68 \%$ & $27 \mathrm{sec}$ & $10 \%$ & $96.00 \%$ & $4.00 \%$ & $42 \mathrm{sec}$ \\
\hline
\end{tabular}

Table 3 demonstrates the TA performance results in the training and testing results. The high TA performance accuracy obtained in the size $70 \%$ training with $97.283 \%$, and $10 \%$ testing with $4 \%$. On the other hand, the lowest performance accuracy results achieved in the $80 \%$ training with $94.215 \%$ and $80 \%$ testing stage with $90 \%$ rate. Figure 3 shown the training and testing results in visualization shape.

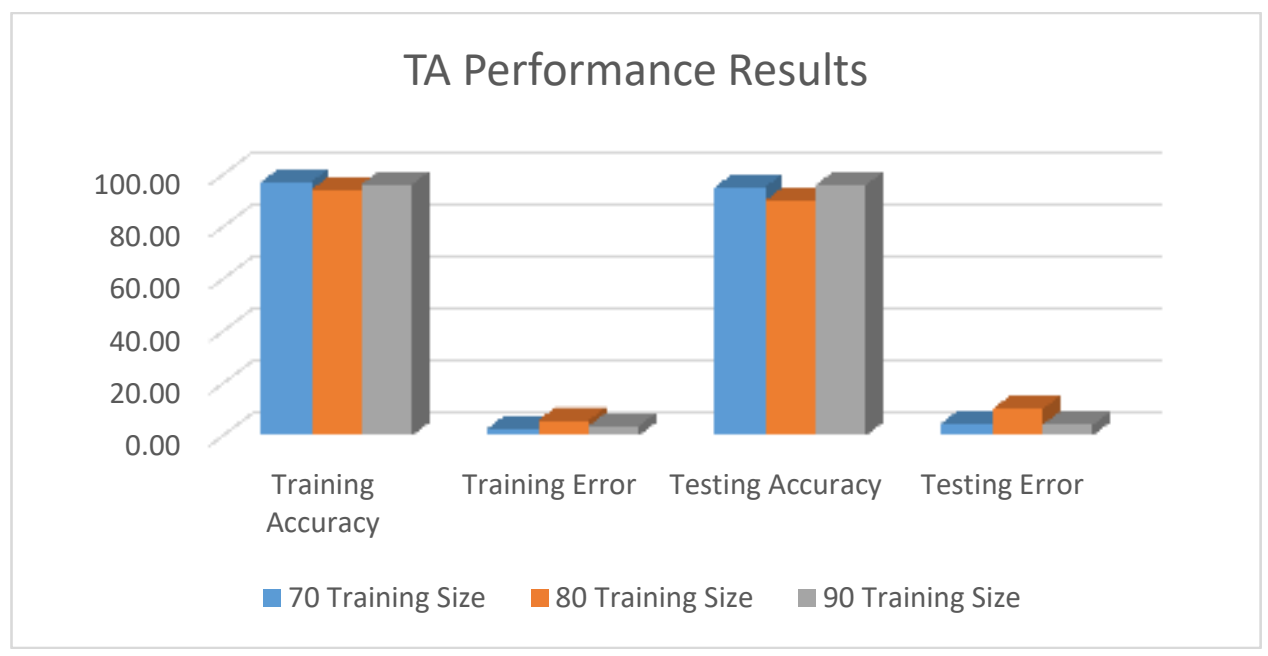

Figure 3. The TA performance results

6. Conclusion. This paper tried to investigate and evaluate the teaching assistant staff to adopt the prediction process based on the data size, time process, accuracy, estimated error factor. The quality of teaching assistant staff was emphasized using artificial neural network algorithms In this study, the experiments conducted based on UCI teaching assistant dataset. The results of the evaluation obtained using with different size in training and testing phases. The deep examinations highlighted that the group of $90 \%$ achieved better results in the prediction accuracy, estimated time, and error factor.

\section{ACKNOWLEDGMENT}

The faculty of computing and information technology at King Abdulaziz University, Saudi Arabia, supported this work. The author would like also to thank the Faculty of Computing and Information Technology at Rabigh (FCITR) for providing the proper research environment. 


\section{REFERENCES}

[1] Yang, J., Jiang, H., \& Zhang, H. (2011). Teaching Assistant Evaluation Based on Support Vector Machines with Parameters Optimization. Information Technology Journal, 10(11), 2140-2146.

[2] Badur, B., \& MARDIKYAN, S. (2011). Analyzing teaching performance of instructors using data mining techniques. Informatics in Education-An International Journal, (Vol 10_2), 245-257.

[3] Bao, Q., Newport, D., Chen, M., Stout, D. B., \& Chatziioannou, A. F. (2009). Performance evaluation of the inveon dedicated PET preclinical tomograph based on the NEMA NU-4 standards. Journal of Nuclear Medicine. 50(3), 401-408.

[4] Baradwaj, B. K., \& Pal, S. (2012). Mining educational data to analyze students' performance. arXiv preprint arXiv:1201.3417.

[5] Yadav, S. K., \& Pal, S. (2012). Data mining: A prediction for performance improvement of engineering students using classification. arXiv preprint arXiv:1203.3832.

[6] Ola, A., \& Pallaniappan, S. (2013). A data mining model for evaluation of instructors' performance in higher institutions of learning using machine learning algorithms. International Journal of Conceptions on Computing and Information Technology, 1(1).

[7] Ahmadi, F., \& Ahmad, M. S. (2013). Data Mining in Teacher Evaluation System using WEKA. International Journal of Computer Applications, 63(10).

[8] Lichman, M. (2013). UCI Machine Learning Repository [http://archive.ics.uci.edu/ml]. Irvine, CA: University of California, School of Information and Computer Science.

[9] Wang, S. J., Mathew, A., Chen, Y., Xi, L. F., Ma, L., \& Lee, J. (2009). Empirical analysis of support vector machine ensemble classifiers. Expert Systems with applications, 36(3), 6466-6476.

[10] Thai-Nghe, N., Busche, A., \& Schmidt-Thieme, L. (2009, November). Improving academic performance prediction by dealing with class imbalance. In Intelligent Systems Design and Applications, 2009. ISDA'09. Ninth International Conference on (pp. 878-883). IEEE.

[11] Gaemperli, O., Schepis, T., Kalff, V., Namdar, M., Valenta, I., Stefani, L., \& Kaufmann, P. A. (2007). Validation of a new cardiac image fusion software for three-dimensional integration of myocardial perfusion SPECT and stand-alone 64-slice CT angiography. European journal of nuclear medicine and molecular imaging, 34(7), 1097-1106.

[12] Jorge, A., Poças, J., \& Azevedo, P. (2002). Post-processing operators for browsing large sets of association rules. In Discovery Science (pp. 251-263). Springer Berlin/Heidelberg.

[13] Aksoezen, M., Daniel, M., Hassler, U., \& Kohler, N. (2015). Building age as an indicator for energy consumption. Energy and Buildings, 87, 74-86.

[14] Elssied, N., Ibrahim, O., \& Osman, A. H. (2014). A novel feature selection based on one-way anova f-test for e-mail spam classification. Research Journal of Applied Sciences, Engineering and Technology, 7(3), 625-638.

[15] Elssied, N. O. F., Ibrahim, O., \& Osman, A. H. (2015). Enhancement of spam detection mechanism based on hybrid $\{\mathrm{k}\}$-mean clustering and support vector machine. Soft Computing, 19(11), 3237-3248. 